\title{
TEE Pocket Manual - Revised Reprint
}

\author{
Leslie Groban, John F. Butterworth IV. Elsevier, Philadelphia PA, 2011. \\ ISBN: 978-1-4557-0588-7.
}

\author{
Jordan Hudson, MD
}

Received: 24 November 2011/ Accepted: 30 November 2011/Published online: 9 December 2011

(C) Canadian Anesthesiologists' Society 2011

This pocket-sized reference manual on transesophageal echocardiography (TEE) was written to facilitate quick access to information on the normal values, variants, calculations, and diagnostic criteria necessary for TEE interpretation and reporting. The manual was not intended as a substitute for more comprehensive echocardiography textbooks, nor does it provide the depth of information included in such texts. Small and lightweight, it fits easily into a lab coat or scrub pocket.

The book is comprised of 21 sections which are separated by colour-coded tabs for rapid access to the information of interest. Included with the manual is a laminated card with a summary of common TEE diagnostic criteria and images: very useful for quick reference. Unlike the first edition, however, the revised edition issued for review does not include access to an online PDF version of the text for access via mobile devices.

The information is presented in point form and is succinct and neatly organized, with liberal use of images and diagrams in most chapters. The basic TEE facts are accurate and as comprehensive as the pocket-sized medium allows. Due to the highly visual nature of TEE, the images are particularly helpful to illustrate key concepts, and despite the small format, the TEE images and illustrations show good clarity and resolution.

Particular attention is paid to the practical aspects of TEE: headings highlight limitations and how to overcome them, as well as surgery-specific imaging requirements and

J. Hudson, MD ( $\bowtie)$

The Ottawa Hospital, University of Ottawa,

Ottawa, ON, Canada

e-mail: jkchudson@gmail.com measurements. This feature may be especially useful for novice perioperative echocardiographers and trainees.

New to this revised reprint are additional chapters on prosthetic valves, three-dimensional (3D) TEE, intracardiac masses, and assessment of the aorta. As well, content for aortic and mitral valves has been divided into assessment of normal, stenotic, and regurgitant valves. The new content covers areas that are essential for clinicians performing TEE in a cardiac surgical environment, particularly with the increasing availability of 3D TEE.

Limitations to this text include a paucity of accompanying images in some chapters, particularly those concerning artifacts and intracardiac masses. Several TEE images used in the text identify both medical centre and date/time of image acquisition, which may pose a risk to patient confidentiality. While most images are referenced, with the exception of the chapter on $3 \mathrm{D}$ TEE, the text is not. This was likely a decision to minimize the book's size, but a link to an online reference page would be a feasible alternative. The number of pages dedicated to content is occasionally unevenly weighted, with sixteen pages concerning diastolic dysfunction and only a single page regarding regional wall motion abnormalities. While the chapter on 3D TEE is a welcome addition to the text, it is rather brief at fewer than five pages. However, the approach used to distribute content and place emphasis on various topics is a highly subjective matter.

The most notable omission from this manual is the absence of accompanying video images, either online or in CD-ROM format. This has become the standard for TEE resources, and it is indispensable when learning an imaging modality which is performed using video loops.

Overall, the authors accomplished their goal of providing a portable reference manual for TEE which facilitates quick and easy access to essential information. In an age 
where many clinicians access information via a mobile device and many TEE resources provide video loops to enhance learning, it may not be the ideal book for all anesthesiologists. However, for residents and fellows or any echocardiographer looking for a pocket-sized paperback reference manual, this book may be a useful adjunct to standard comprehensive TEE texts.

Competing interests None declared. 